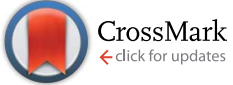

Cite this: RSC Adv., 2016, 6, 86955

Received 24th August 2016 Accepted 7th September 2016

DOI: $10.1039 / c 6 r a 21296 d$

www.rsc.org/advances

\section{On the stability of surfactant-stabilised few-layer black phosphorus in aqueous media $\uparrow$}

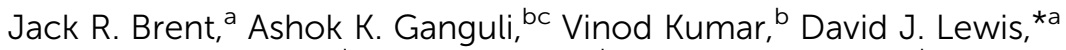 \\ Paul D. McNaughter, ${ }^{\text {d }}$ Paul O'Brien, ${ }^{\text {ad }}$ Priyanka Sabherwal ${ }^{\mathrm{b}}$ \\ and Aleksander A. Tedstone ${ }^{d}$
}

Surfactant-assisted exfoliation routes to few-layer black phosphorus in aqueous media have recently been reported. The stability of such few-layer black phosphorus has been studied using a range of spectroscopic techniques. The material is meta-stable in aqueous media, degrading over time mainly to phosphoric acids.

Graphene and other two-dimensional (2D) materials have revolutionised materials science. ${ }^{1}$ The properties displayed by $2 \mathrm{D}$ materials are markedly different to their bulk counterparts. ${ }^{2}$ For instance, graphene shows ballistic carrier mobility and is a superb conductor of electricity, ${ }^{3,4}$ whilst monolayer molybdenum disulfide becomes a direct bandgap semiconductor with strong photoluminescence. ${ }^{5}$ Hence, 2D materials have attracted great interest over the past decade and have the potential to revolutionise a range of fields. ${ }^{6}$

2D materials may be synthesised in several different ways. Micromechanical pinacoidal cleavage of bulk crystals (the Scotch Tape method), made popular by Geim and co-workers, ${ }^{7}$ has been the most popular of all the processing techniques; but is strictly limited to demonstrative studies. More useful perhaps is the large scale growth of polycrystalline wafers of 2D materials by chemical vapour deposition. ${ }^{8}$ One of the simplest ways that these materials can be produced, and additionally in solution, is by ultrasonic cavitation, which has been applied to most crystals that show basal cleavage. ${ }^{9-13}$ Shear exfoliation in surfactants has recently been reported, and can potentially be carried out on scale. ${ }^{14}$

Recently, there has been interest in elemental 2D synthetic allotropes which can show various properties complimentary

${ }^{a}$ School of Materials, University of Manchester, Oxford Road, M13 9PL, UK. E-mail: david.lewis-4@manchester.ac.uk; paul.o'brien@manchester.ac.uk

${ }^{b}$ Institute of Nano Science \& Technology (INST), Habitat Centre, Phase-10, Sec-64, Mohali-160062, Punjab, India

'Department of Chemistry, Indian Institute of Technology Delhi, Hauz Khas, New Delhi-110016, India

${ }^{d}$ School of Chemistry, University of Manchester, Oxford Road, M13 9PL, UK

$\dagger$ Electronic supplementary information (ESI) available: Materials, instrumentation and detailed exfoliation procedure. See DOI: 10.1039/c6ra21296d to graphene. Silicene (2D silicon), ${ }^{15}$ borophene (2D boron) ${ }^{16}$ germanene (2D germanium) ${ }^{17}$ and stanene $(2 \mathrm{D} \text { tin })^{18}$ have all either been reported or predicted. ${ }^{19}$ One such elemental analogue, phosphorene (2D black phosphorus), has attracted attention due to its high p-type carrier mobility and its layerdependent band gap that spans the range between graphene (negligible band gap) and 2D $\mathrm{MoS}_{2}(c a .1 .5 \mathrm{eV}){ }^{20} \mathrm{~A}$ range of functional devices based on this material have been produced including transistors. ${ }^{21}$ Phosphorene is produced by exfoliation of the layered black phosphorus ${ }^{22}$ allotrope. Synthetic routes to the material include micromechanical cleavage ${ }^{20}$ and solution phase exfoliation. ${ }^{23-25}$ CVD routes to this material remain elusive.

Questions remain regarding the stability of phosphorene, or few-layer black phosphorus (FL-BP), that may stymie its eventual exploitation. The major problem is that the material is reactive to oxygen-containing species due to the strength of the $\mathrm{P}=\mathrm{O}$ bond. Studies have shown that water in particular causes degradation of FL-BP in solution, as well as in exposed flakes. The solution-exfoliated material is more stable than micromechanically exfoliated material. Strategies to prevent chemical degradation of FL-BP have included coating with oxides, ${ }^{26}$ entombment within other $2 \mathrm{D}$ materials by vertical stacking of flakes ${ }^{27}$ and, in the case of solution exfoliated material, physical barriers to atmospheric ingress ${ }^{28}$ and the use of sterically hindered solvents such as cyclohexyl-2-pyrrolidone (CHP). ${ }^{25}$

Very recently, Hersam and co-workers introduced the concept of surfactant-assisted ultrasonic exfoliation to produce dispersions of FL-BP in aqueous media. ${ }^{29}$ In this study, the FLBP sols were produced by ultrasonication of layered bulk black phosphorus in degassed water containing $2 \% \mathrm{w} / \mathrm{v}$ sodium dodecyl sulfate (SDS) surfactant. This presents an elegant solution-based strategy to protect FL-BP from degradation in theory. The authors claim that the solutions are stable, yet no stability study over time was presented. Ultimately the removal of organic solvents from processing is a well-principled aim in terms of scale up due to the cost of NMP and for environmental concerns and thus merits investigation. In this paper we present 
a multi-spectroscopic temporal stability study of surfactantexfoliated FL-BP sols in aqueous media.

We exfoliated FL-BP using 1\% w/v Triton X-100 (TX-100, $\mathrm{C}_{14} \mathrm{H}_{22} \mathrm{O}\left(\mathrm{C}_{2} \mathrm{H}_{4} \mathrm{O}\right)_{n}$ where $\left.n=9-10\right)$ surfactant in water. In a sealed vial, the degassed surfactant solution $(15 \mathrm{~mL})$ was added to bulk BP (100 mg) and the vial was flushed with argon, closed and sealed with Parafilm ${ }^{\circledR}$. Suspensions were sonicated at $298 \mathrm{~K}$ in an Elmasonic P $70 \mathrm{H}$ bench-top ultrasonic bath (820 $\mathrm{W}$ across four horns) operating at $37 \mathrm{kHz}$ and $30 \%$ power. After $36 \mathrm{~h}$ the dispersions were centrifuged at $1500 \mathrm{rpm}$ for $45 \mathrm{~min}$ and the supernatant removed. Further $10 \mathrm{~mL}$ of surfactant solution was added to the pellet of FL-BP which was sonicated again for $12 \mathrm{~h}$ under the same conditions. The dispersion was centrifuged again and the supernatant was added to the previously collected dispersion. Our developed procedure, reported previously, produces FL-BP of sub-20 nm thickness and $c a$. 100$200 \mathrm{~nm}$ in side. ${ }^{30}$

The stability of the nanosheets, was assessed by the optical absorbance at $465 \mathrm{~nm}$ over time. The degradation of the flakes was fitted to an empirical decay function used previously by Coleman and co-workers for FL-BP nanosheets: ${ }^{25}$

$$
A_{t} / A_{0}=A_{\text {stable }}+A_{\text {unstable }} \mathrm{e}^{-t / \tau}
$$

where $A_{t}$ is the relative absorbance at $465 \mathrm{~nm}$ at time $t$ compared to that at the start $\left(A_{0}\right)$, represented by the sum of the absorbance of intact, stable BP nanosheets $\left(A_{\text {stable }}\right)$, and a term that represents the degradation of the sheets $\left(A_{\text {unstable }} \mathrm{e}^{-t / \tau}\right)$, where $\tau$ is observed decay constant of the nanosheets. Thus by recording the UV-Vis absorbance at $465 \mathrm{~nm}$ over $600 \mathrm{~h}$, it was found that the observed decay constant of the exfoliated nanosheets (in 1\%

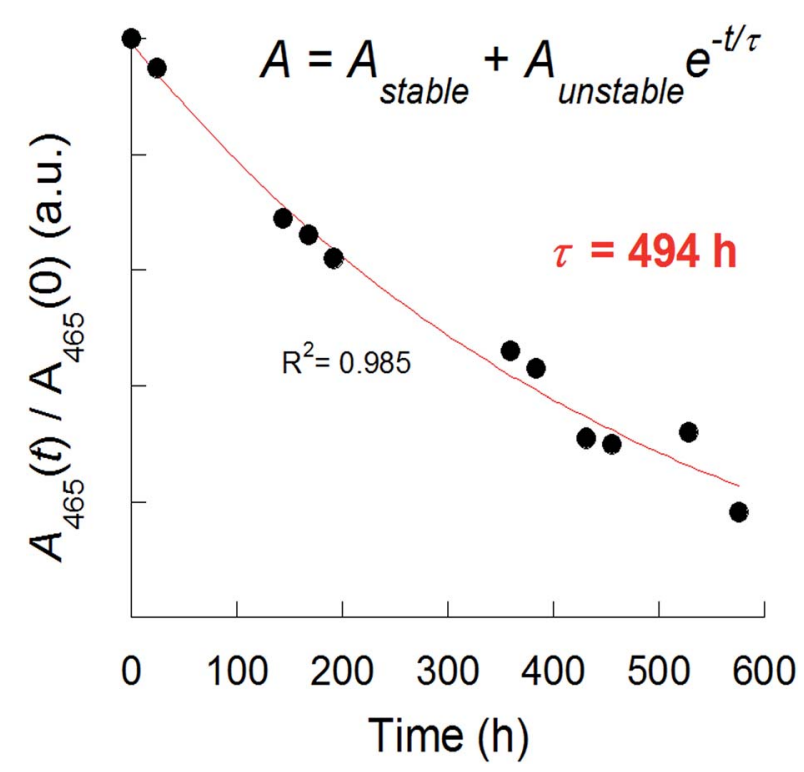

Fig. 1 UV-Vis absorbance spectroscopy time study of the stability of FL-BP nanosheets in $1 \% \mathrm{w} / \mathrm{V}$ aqueous Triton $\mathrm{X}-100$. The change in absorbance at $465 \mathrm{~nm}$ is used as an indicator of the amount of black phosphorus remaining in solution and can be fitted to the empirical function in the inset. The mean observed lifetime of the nanosheets in solution is estimated to be almost $500 \mathrm{~h}$. w/v aqueous Triton X-100 solution) was $\tau=494 \mathrm{~h}$ (Fig. 1). The total percentage degradation of the sheets in solution was around $80 \%$ over this time, estimated from the total decrease in absorbance of the sol. FL-BP exfoliated in $\mathrm{N}$-cyclohexyl-2pyrrolidone (CHP) have decay constants in the range 115 to $350 \mathrm{~h}$, and the total degradation of CHP-exfoliated BP is reported to be $25 \%$, and is believed to be limited by the amount of water in the organic solvent. It is clear from the observed decay constant we measure here then that the surfactant plays a crucial role in slowing down the degradation process, protecting the surface and edges of the nanosheets from water, possibly by micelle formation. We postulate that the Triton X100 head group, consisting of ${ }^{t} \mathrm{Bu}\left(\mathrm{CH}_{2}\right)_{2} \mathrm{Ph}-\mathrm{R}$ which is highly non-polar, is able to form an effective surface-bound layer protecting the BP from degradation by the surrounding water. The polar polyethylene oxide chains of the surfactant extend into the aqueous media. Lewis et al. have demonstrated previously that surfactants are useful for both the dispersion of main group oxide nanoparticles, as well as enhancement of the photoluminescence emission of surface-bound luminophores that are quenched by water and oxygen. ${ }^{31}$

We further probed the stability of the BP nanosheets in aqueous media by considering solutions with the nanosheets removed by centrifugation followed by a polish with membrane ultrafiltration to leave clear, non-hazy, mother liquors. Inductively coupled plasma optical emission spectroscopy (ICP-OES) on these mother liquors revealed that the decrease in the absorption at $465 \mathrm{~nm}$ observed over time i.e. the degradation of FL-BP nanosheets, is accompanied by the release of phosphorus species into solution (Fig. 2). The amount of phosphorus detected by ICP-OES rises to a plateau at around in around 500 $\mathrm{h}$, suggesting that there is a critical point at which the nanosheets cannot degrade any further, presumably where a critical micelle concentration is reached. There is no possibility that these phosphorus species remain bound to the surface of the FL-BP sheets as these are removed by the centrifugation-

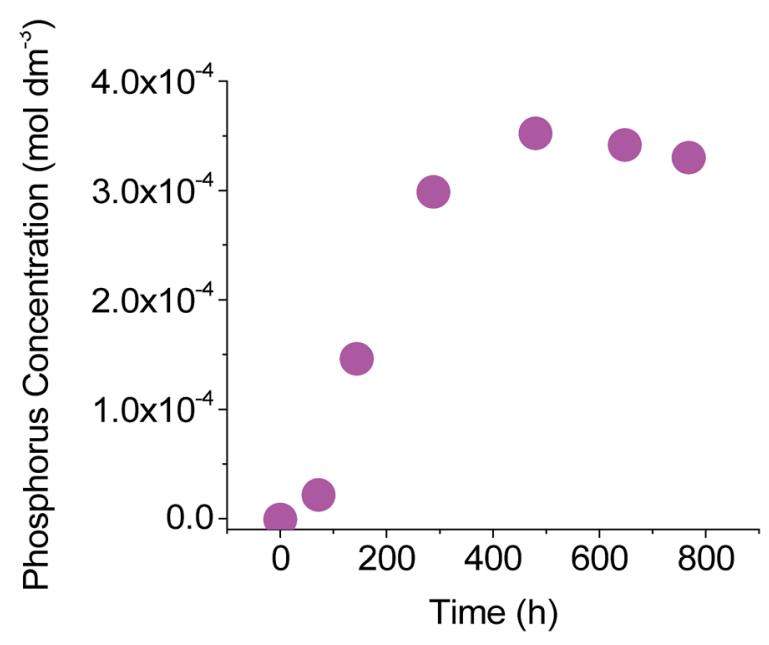

Fig. 2 ICP-OES time study of the absolute concentration of phosphorus released into solution from degradation of the FL-BP nanosheets in $1 \% \mathrm{w} / \mathrm{v}$ aqueous Triton $\mathrm{X}-100$, showing a plateau-like profile. 


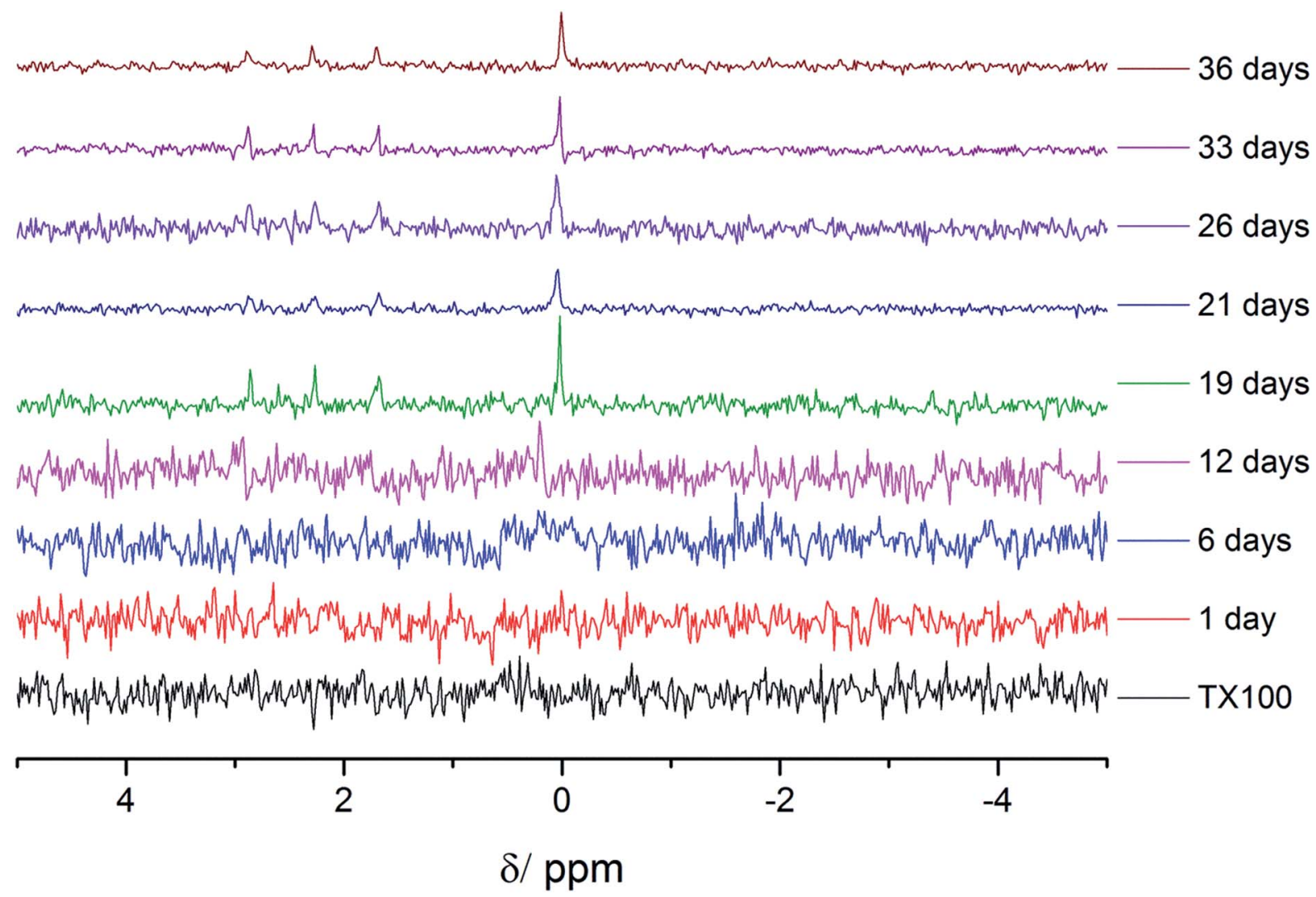

Fig. 3 Time dependence of the ${ }^{31} \mathrm{P}$ spectrum of the phosphorus species released into solution from degradation of FL-BP sheets in $1 \%$ w/V aqueous Triton X-100 over a period of 36 days ( $864 \mathrm{~h}$ ). The black trace labelled TX100 is the ${ }^{31} \mathrm{P}$ NMR spectrum of $1 \% \mathrm{w} / \mathrm{v}$ aqueous Triton X-100, which contains no phosphorus species.

ultrafiltration procedure we employ and hence we are confident that we are looking solely at breakdown products that have been released into solution.

The chemical identity of the species released was probed by ${ }^{31} \mathrm{P}$ nuclear magnetic resonance (NMR) spectroscopy using $\mathrm{H}_{3} \mathrm{PO}_{4}$ as a standard at 0 ppm (Fig. 3). The peaks which appear at $c a$. 1-3 ppm were assigned to orthophosphate, $\left(\mathrm{PO}_{4}{ }^{3-}\right)$. Hence, we can confidently state that the sheets degrade to phosphates in solution from hydrolytic mechanisms, and that the nanosheets are meta-stable when dispersed in aqueous media and thus have a 'shelf life'.

In conclusion, we have shown that FL-BP nanosheets can be stabilised in aqueous media by the use of surfactants, however, there is some relatively slow degradation of the freshly formed material to form free $\mathrm{PO}_{x}$ phosphate species. Care should hence be taken when using FL-BP sols to account for the possibility of this degradation over time. It seems however, that a more stable form can be reached in which further degradation is limited and in this sense the sols appear to be meta-stable. This will be important when, for instance, considering the biological applications of phosphorene, some of which have recently been reported. ${ }^{32}$

\section{Acknowledgements}

P. O. B., P. D. M. and J. R. B. would like to thank the Parker family and EPSRC (EP/K009710/1) for funding. V. K. and P. S. acknowledge INST PDF, CSIR-JRF, and DST, Govt. of India for funding. A. K. G. thanks DST, Govt. of India for financial support to INST, Mohali, India. Some of the equipment used in this study were provided by EPSRC (Core Capability in Chemistry, EPSRC grant number EP/K039547/1). Additional data associated with this paper is available from the University of Manchester's Pure repository. The authors would also like to thank Paul Lythgoe (University of Manchester) for running ICPOES and Dr Ralph Adams (University of Manchester) for guidance with NMR measurements.

\section{Notes and references}

1 A. K. Geim and K. S. Novoselov, Nat. Mater., 2007, 6, 183-191. 2 Q. H. Wang, K. Kalantar-Zadeh, A. Kis, J. N. Coleman and M. S. Strano, Nat. Nanotechnol., 2012, 7, 699-712.

3 K. S. Novoselov, A. K. Geim, S. V. Morozov, D. Jiang, M. I. Katsnelson, I. V. Grigorieva, S. V. Dubonos and A. A. Firsov, Nature, 2005, 438, 197-200.

4 K. S. Novoselov, A. K. Geim, S. V. Morozov, D. Jiang, M. I. Katsnelson, I. V. Grigorieva, S. V. Dubonos and A. A. Firsov, Science, 2004, 306, 666-669.

5 K. F. Mak, C. Lee, J. Hone, J. Shan and T. F. Heinz, Phys. Rev. Lett., 2010, 105, 136805.

6 P. Miro, M. Audiffred and T. Heine, Chem. Soc. Rev., 2014, 43, 6537-6554. 
7 K. S. Novoselov, D. Jiang, F. Schedin, T. J. Booth, V. V. Khotkevich, S. V. Morosov and A. K. Geim, Proc. Natl. Acad. Sci. U. S. A., 2005, 102, 10451-10453.

8 A. Reina, X. T. Jia, J. Ho, D. Nezich, H. B. Son, V. Bulovic, M. S. Dresselhaus and J. Kong, Nano Lett., 2009, 9, 30-35.

9 J. N. Coleman, M. Lotya, A. O'Neill, S. D. Bergin, P. J. King, U. Khan, K. Young, A. Gaucher, S. De, R. J. Smith, I. V. Shvets, S. K. Arora, G. Stanton, H.-Y. Kim, K. Lee, G. T. Kim, G. S. Duesberg, T. Hallam, J. J. Boland, J. J. Wang, J. F. Donegan, J. C. Grunlan, G. Moriarty, A. Shmeliov, R. J. Nicholls, J. M. Perkins, E. M. Grieveson, K. Theuwissen, D. W. McComb, P. D. Nellist and V. Nicolosi, Science, 2011, 331, 568-571.

10 Y. Hernandez, V. Nicolosi, M. Lotya, F. M. Blighe, Z. Sun, S. De, I. T. McGovern, B. Holland, M. Byrne, Y. K. Gun'Ko, J. J. Boland, P. Niraj, G. S. Duesberg, S. Krishnamurthy, R. Goodhue, J. Hutchison, V. Scardaci, A. C. Ferrari and J. N. Coleman, Nat. Nanotechnol., 2008, 3, 563-568.

11 A. O'Neill, U. Khan and J. N. Coleman, Chem. Mater., 2012, 24, 2414-2421.

12 N. Al-Dulaimi, E. A. Lewis, D. J. Lewis, S. K. Howell, S. J. Haigh and P. O'Brien, Chem. Commun., 2016, 52, 7878-7881.

13 J. R. Brent, D. J. Lewis, T. Lorenz, E. A. Lewis, N. Savjani, S. J. Haigh, G. Seifert, B. Derby and P. O'Brien, J. Am. Chem. Soc., 2015, 137, 12689-12696.

14 K. R. Paton, E. Varrla, C. Backes, R. J. Smith, U. Khan, A. O'Neill, C. Boland, M. Lotya, O. M. Istrate, P. King, T. Higgins, S. Barwich, P. May, P. Puczkarski, I. Ahmed, M. Moebius, H. Pettersson, E. Long, J. Coelho, S. E. O'Brien, E. K. McGuire, B. M. Sanchez, G. S. Duesberg, N. McEvoy, T. J. Pennycook, C. Downing, A. Crossley, V. Nicolosi and J. N. Coleman, Nat. Mater., 2014, 13, 624-630.

15 L. Tao, E. Cinquanta, D. Chiappe, C. Grazianetti, M. Fanciulli, M. Dubey, A. Molle and D. Akinwande, Nat. Nanotechnol., 2015, 10, 227-231.

16 A. J. Mannix, X.-F. Zhou, B. Kiraly, J. D. Wood, D. Alducin, B. D. Myers, X. Liu, B. L. Fisher, U. Santiago, J. R. Guest, M. J. Yacaman, A. Ponce, A. R. Oganov, M. C. Hersam and N. P. Guisinger, Science, 2015, 350, 1513-1516.

17 M. E. Dávila, L. Xian, S. Cahangirov, A. Rubio and G. L. Lay, New J. Phys., 2014, 16, 095002.

18 F.-f. Zhu, W.-j. Chen, Y. Xu, C.-l. Gao, D.-d. Guan, C.-h. Liu, D. Qian, S.-C. Zhang and J.-f. Jia, Nat. Mater., 2015, 14, 1020-1025.
19 S. Balendhran, S. Walia, H. Nili, S. Sriram and M. Bhaskaran, Small, 2015, 11, 640-652.

20 H. Liu, A. T. Neal, Z. Zhu, Z. Luo, X. Xu, D. Tománek and P. D. Ye, ACS Nano, 2014, 8, 4033-4041.

21 L. Li, Y. Yu, G. J. Ye, Q. Ge, X. Ou, H. Wu, D. Feng, X. H. Chen and Y. Zhang, Nat. Nanotechnol., 2014, 9, 372-377.

22 R. Hultgren, N. S. Gingrich and B. E. Warren, J. Chem. Phys., 1935, 3, 351-355.

23 J. R. Brent, N. Savjani, E. A. Lewis, S. J. Haigh, D. J. Lewis and P. O'Brien, Chem. Commun., 2014, 50, 13338-13341.

24 P. Yasaei, B. Kumar, T. Foroozan, C. Wang, M. Asadi, D. Tuschel, J. E. Indacochea, R. F. Klie and A. SalehiKhojin, Adv. Mater., 2015, 27, 1887-1892.

25 D. Hanlon, C. Backes, E. Doherty, C. S. Cucinotta, N. C. Berner, C. Boland, K. Lee, A. Harvey, P. Lynch, Z. Gholamvand, S. Zhang, K. Wang, G. Moynihan, A. Pokle, Q. M. Ramasse, N. McEvoy, W. J. Blau, J. Wang, G. Abellan, F. Hauke, A. Hirsch, S. Sanvito, D. D. O'Regan, G. S. Duesberg, V. Nicolosi and J. N. Coleman, Nat. Commun., 2015, 6, 8563.

26 J. D. Wood, S. A. Wells, D. Jariwala, K.-S. Chen, E. Cho, V. K. Sangwan, X. Liu, L. J. Lauhon, T. J. Marks and M. C. Hersam, Nano Lett., 2014, 14, 6964-6970.

27 Y. Cao, A. Mishchenko, G. L. Yu, E. Khestanova, A. P. Rooney, E. Prestat, A. V. Kretinin, P. Blake, M. B. Shalom, C. Woods, J. Chapman, G. Balakrishnan, I. V. Grigorieva, K. S. Novoselov, B. A. Piot, M. Potemski, K. Watanabe, T. Taniguchi, S. J. Haigh, A. K. Geim and R. V. Gorbachev, Nano Lett., 2015, 15, 4914-4921.

28 J. Kang, J. D. Wood, S. A. Wells, J. Lee, X. Liu, K. Chen and M. C. Hersam, ACS Nano, 2015, 9, 3596-3604.

29 J. Kang, S. A. Wells, J. D. Wood, J.-H. Lee, X. Liu, C. R. Ryder, J. Zhu, J. R. Guest, C. A. Husko and M. C. Hersam, Proc. Natl. Acad. Sci. U. S. A., 2016, DOI: 10.1073/pnas.1602215113.

30 V. Kumar, J. R. Brent, M. Shorie, H. Kaur, G. Chadha, A. G. Thomas, E. A. Lewis, A. P. Rooney, L. Nguyen, X. L. Zhong, M. G. Burke, S. J. Haigh, A. S. Walton, P. D. McNaughter, A. A. Tedstone, N. Savjani, C. A. Muryn, P. O'Brien, A. K. Ganguli, D. J. Lewis and P. Sabherwal, ACS Appl. Mater. Interfaces, 2016, 8, 22860-22868.

31 D. J. Lewis, V. Dore, N. J. Rogers, T. K. Mole, G. B. Nash, P. Angeli and Z. Pikramenou, Langmuir, 2013, 29, 1470114708.

32 H. Wang, X. Yang, W. Shao, S. Chen, J. Xie, X. Zhang, J. Wang and Y. Xie, J. Am. Chem. Soc., 2015, 137, 11376-11382. 